\title{
Criterios e Instrumentos para la Admisión en los Estudios de Doctorado
}

\section{Francisco Álvarez Montero ${ }^{1}$, Ambrocio Mojardín Heráldez ${ }^{2}$, Carmen Audelo López ${ }^{1}$}

${ }^{1}$ Facultad de Ciencias de la Educación, Universidad Autónoma de Sinaloa ${ }^{2}$ Facultad de Psicología, Universidad Autónoma de Sinaloa

\section{México}

Correspondencia: Francisco Álvarez-Montero. Facultad de Ciencias de la Educación, Universidad Autónoma de Sinaloa, C/Platón 856, Villa Universidad, C.P. 80010, Culiacán. México.

E-mail: francisco_alvarez_montero@uas.edu.mx

(C) Education \& Psychology I+D+i and Editorial EOS (Spain) 


\section{Resumen}

Los estudios de posgrado, en particular los de doctorado, están orientados a la formación de investigadores capaces de hacer contribuciones originales dentro de un área de conocimiento. Sin embargo, las tasas de abandono o deserción indican que lograr este objetivo no es tarea fácil. Los estudios realizados indican que existen factores de comportamiento, positivos y negativos, que influencian la obtención del grado de doctor. En México, a diferencia de los países anglosajones, estos aspectos han sido muy poco estudiados. En este artículo se analiza la relación entre el éxito académico y los instrumentos comúnmente utilizados para justificar el ingreso a los estudios de licenciatura (EXANI-II) y a los de posgrado (EXANI-III) en este país. Adicionalmente, se introducen una serie de constructos psicológicos medibles, diferentes a los que comprenden los EXANI, que pueden utilizarse para la admisión a estudios de Doctorado y aumentar la certidumbre en los índices de titulación a través de tesis originales defendidas en los tiempos establecidos para ello.

Palabras Clave: EXANI-II, EXANI-III, coeficiente intelectual, eficiencia terminal, éxito académico, validez predictiva, auto-sabotaje, valor, autodisciplina, propósito, creatividad. 


\title{
Criteria and Instruments for Doctoral Program Admissions
}

\begin{abstract}
Graduate studies, and in particular doctoral ones, pursue the development of scientific researchers able to make original contributions in a specific area of knowledge. However, attrition rates indicate that achieving this goal is not easy. The available evidence indicates that there are behavioral factors, positive and negative, that influence obtaining a doctoral degree. Unlike in other western nations, such as the USA, these factors have not been studied in Mexico. In particular, this article analyzes the relationship between academic success and the instruments commonly used to decide admission to undergraduate (EXANI-II) and postgraduate studies (EXANI-III) in Mexico. Additionally, a number of measurable psychological constructs are introduced. These constructs are different from those comprising the EXANI and can be used for admission to doctoral studies, to reduce attrition rates and increase the certainty about the timely completion of Doctoral dissertations.
\end{abstract}

Keywords: EXANI-II, EXANI-III, intellectual quotient, graduation rate, academic achievement, predictive validity, self-sabotage, grit, self-discipline, achievement goals, creativity. 


\section{Introducción}

Desde 1970 se ha observado en México un crecimiento en la matrícula de Educación Ssuperior, siendo el posgrado el nivel que más incremento presenta (Esquivel \& Rojas, 2005). Adicionalmente, desde inicios de la década de 1980, las políticas educativas mexicanas están formuladas a partir de conceptos como la excelencia académica, calidad de la educación y eficiencia terminal (Sevilla, Martín \& Guillermo, 2009). Auspiciadas por el Banco Mundial y el Fondo Monetario Internacional, estas ideas tienen su origen en el flujo y manejo eficiente de materiales y mano de obra, para la producción de artículos industriales de calidad, en los tiempos establecidos para ello (Kannan \& Tan, 2005; Watson, Blackstone \& Gardiner, 2007). Por tanto, no buscan logros en los aspectos cognitivos (Greeno, Collins \& Resnick, 1996), pedagógicos (Leach \& Moon, 2008), filosóficos (Phillips et al., 2010), o de diseño (Gagne et al., 2005) de la Educación.

De todos estos conceptos, quizá uno de los más importantes para los posgrados mexicanos (i.e., Maestría o Doctorado) sea el denominado eficiencia terminal, también conocido como éxito académico (Martínez et al., 2003; Sevilla, Martín \& Guillermo, 2009). Buena prueba de ello es que el Consejo Nacional de Ciencia y Tecnología (CONACYT), a través del Padrón Nacional de Posgrados de Calidad (PNPC), establece que de acuerdo a su nivel de calidad, los posgrados deben de alcanzar las siguientes tasas de eficiencia por cohorte (CONACYT, 2013): a) para posgrados en desarrollo (40\%); b) para posgrados consolidados $(50 \%)$ y c) para posgrados de competencia internacional (60\%).

Las definiciones de eficiencia terminal abundan (de los Santos, 2003; Colonia, 2010). Sin embargo, todas ellas hacen referencia al número de estudiantes o alumnos que obtienen el grado académico, en los tiempos establecidos por los planes y programas de estudio y, con los estándares de calidad definidos por la institución. Para los posgrados mexicanos, en particular para los que pertenecen al PNPC, una de las implicaciones del uso del concepto de eficiencia terminal, como variable medidora de su funcionamiento, es que se debe cuidar el proceso de selección o admisión, para garantizar que los aspirantes admitidos obtengan el grado, en el lapso establecido, y asegurar así, la permanencia en el PNPC (Sevilla, Martín \& Guillermo, 2009; Solís, 2009). Sin embargo, en México este tema ha recibido poca atención. Prueba de ello es que en el último libro publicado por el Consejo Mexicano de Estudios de Posgrado 
(COMEPO), de 41 artículos sólo 2 artículos (Maya, Chávez \& Apolinar, 2012; Pérez, Serna \& Barriga, 2012) abordan el proceso de admisión al posgrado y lo hacen de manera tangencial. En particular, sólo mencionan los criterios de admisión (i.e., puntuación mínima en el Examen Nacional de Ingreso a Posgrado o EXANI-III) promedio de Licenciatura y calificación en el examen TOEFL (Test of English as a Foreign Language). No hay en estas publicaciones, una revisión de la literatura o un análisis estadístico, que ayude a determinar la relación y el impacto de estos criterios sobre los conceptos de eficiencia terminal, promedio general en el posgrado, producción científica, u otras variables importantes para los posgrados.

Mientras que los procesos de admisión al posgrado, sus criterios y, el impacto que estos tienen es un asunto poco abordado por la comunidad científica mexicana, en los Estados Unidos de América (EUA) este fenómeno sí ha sido estudiado. En particular, la utilización y el impacto de los denominados exámenes estandarizados de admisión (Kuncel, Hezlett \& Ones, 2001; Kuncel, Hezlett \& Ones, 2004; Kuncel \& Hezlett, 2007; Kuncel, Wee, Serafin, \& Hezlett, 2010), como principal criterio de admisión a las Instituciones de Educación Superior (IES), ha sido objeto de múltiples análisis y debates desde principios del siglo XX (Kaufman, 2013).

Los resultados de los meta-análisis más recientes (Kuncel \& Hezlett, 2007; Kuncel et al., 2010) indican que de los exámenes estandarizados que se aplican en los EUA, el Graduate Record Examinations (GRE-T), el Graduate Management Admission Test (GMAT), y el Miller Analogies Test (MAT), son los que mejor predicen la productividad científica, el número de citas y la obtención del grado de los estudiantes, con correlaciones que van desde 0.120 hasta 0.220 . Correlaciones que, aunque positivas, siguen siendo bajas. Por ello, ha surgido un movimiento científico con el objetivo de completar el rompecabezas psicológico del éxito académico, mediante la identificación y análisis de otros criterios de admisión, distintos de los exámenes estandarizados, que al mismo tiempo, tengan un impacto menos adverso para aspirantes provenientes de minorías étnicas o de un estrato socioeconómico bajo (Atkinson \& Geiser, 2009; Busato et al., 2000; Chamorro-Premuzic \& Furnham, 2003; Duckworth et al., 2007; Kaufman, 2010; Kyllonen \& Poropat, 2009; Kyllonen, Walters \& Kaufman, 2005; Sternberg Bonney, Gabora, \& Merrifield, 2012; Tomsho, 2009). 


\section{Objetivos}

Por lo anterior, este artículo tiene dos objetivos. Primero, analizar a partir de una revisión de la literatura, el nivel de validez predictiva de los exámenes estandarizados que realiza el Centro Nacional de Evaluación para la Educación Superior (CENEVAL) para la admisión en Licenciatura (i.e., EXANI-II) y Posgrado (EXANI-III) en México. Segundo, establecer un mínimo de características o cualidades individuales, para aspirantes a estudios de Doctorado, distintas a las que se consideran en los exámenes antes mencionados, que se relacionen con el éxito en el posgrado (i.e., en particular con la obtención del grado académico), que sean medibles y no vayan en detrimento de los aspirantes de minorías étnicas y estratos socioeconómicos bajos.

El resto de este artículo está organizado de la siguiente forma. En primer lugar, se analiza la validez predictiva de los EXANI II y III a través de la literatura existente sobre el tema. En segundo lugar, se establecen los objetivos principales de los estudios de Doctorado y, se describen dos modelos explicativos del éxito en éstos, subrayando la importancia que en ambos modelos tienen las características individuales de los doctorandos. En cuarto lugar, se presentan y se justifican las características individuales más estudiadas en la literatura y que más relación tienen con los objetivos de un programa doctoral. Por último, se presentan algunas conclusiones y el trabajo futuro.

\section{Los exámenes estandarizados de admisión}

Antes de analizar la literatura existente acerca de la validez predictiva de los exámenes estandarizados de admisión a Licenciatura y Posgrado utilizados en México, es necesario responder a las siguientes preguntas: a) ¿cuál es el origen de las pruebas estandarizadas? b) ¿qué miden? c) ¿sobre qué criterios estadísticos se han construido? y d) ¿qué personas son consideradas como dotadas o talentosas, con respecto a los resultados de este tipo de pruebas?

La utilización de exámenes para determinar la admisión o rechazo de una persona, a lo que Lohman (2005) denomina "oportunidad educativa", no es nueva. En México, este tipo de exámenes se empezó a utilizar desde 1994, para justificar la admisión o rechazo a estudios de bachillerato (Hernández, 2007). En EUA, según Kaufman (2013), estas pruebas se han venido utilizando desde 1911. Su propósito original era determinar la edad mental de una persona (Boake, 2002), sin que esto implicara detectar su nivel absoluto de inteligencia, o hacer pre- 
dicciones sobre el futuro académico o laboral de un aspirante o candidato. Sin embargo, estas pruebas terminaron siendo utilizadas como instrumento desde el cual se justificaban el rechazo y castigo de los miembros "indeseables" o "mentalmente débiles" de la sociedad estadunidense (Kaufman, 2013).

En particular, este tipo de exámenes miden uno o más de los siguientes dominios o habilidades cognitivas: razonamiento, habilidad espacial, memoria, velocidad de procesamiento y vocabulario (Deary, Penke \& Johnson, 2010). La medición de estas habilidades implica la utilización de la memoria de trabajo (Gray \& Thompson, 2004; Jaeggi, Buschkuehl, Jonides \& Perrig, 2008; Kaufman, 2013), que es una red o sistema neural para mantener la información activa en la mente (almacenamiento) y manipularla (funcionamiento ejecutivo) a pesar del potencial de distracción o interferencia (atención). Consecuentemente, como Colom, Rebollo, Palacios, Juan y Kyllonen (2004) subrayan, este tipo de pruebas no mide conocimientos específicos, ni habilidades o estrategias para la resolución de problemas, sino las diferencias que existen entre individuos a la hora de procesar información.

Debido a que este tipo de exámenes están influenciados por la escala de inteligencia de Wechsler-Bellevue (Boake, 2002), cosifican un conjunto de decisiones estadísticas arbitrarias. En términos de Kaufman (2013), la primera de ellas es la selección del coeficiente intelectual (CI) promedio de una persona. Se escogió el número 100 debido a que éste era el más común en la fórmula original de Terman (1917), para el cálculo del CI. Este valor es equivalente a la media teórica para los EXANI II y III (CENEVAL, 2013a; CENEVAL, 2013b), e inclusive para el Examen General de Egreso de Licenciatura o EGEL (López \& Flores, 2006). La segunda decisión es la utilización del concepto de desviación estándar, simplemente porque permite a los examinadores colocar los CI en la campana o curva de Gauss, que representa una distribución normal. La tercera y última decisión fue utilizar 15 como la desviación estándar. Esto se debió a que este número es fácilmente divisible y representa la edad en la que Terman y Merrill (1937) consideraban que el CI dejaba de crecer ${ }^{1}$. Con esto, la probabilidad de que en una distribución normal, una observación alcance una desviación estándar es de 0.68. Es decir, el 68\% de la población humana obtendrá un CI entre 85 y 115 (ver figura 1).

\footnotetext{
${ }^{1}$ Según Kaufman (2013), este número es una conjetura, pues Terman y Merrill nunca examinaron a nadie mayor de dieciocho años.
} 


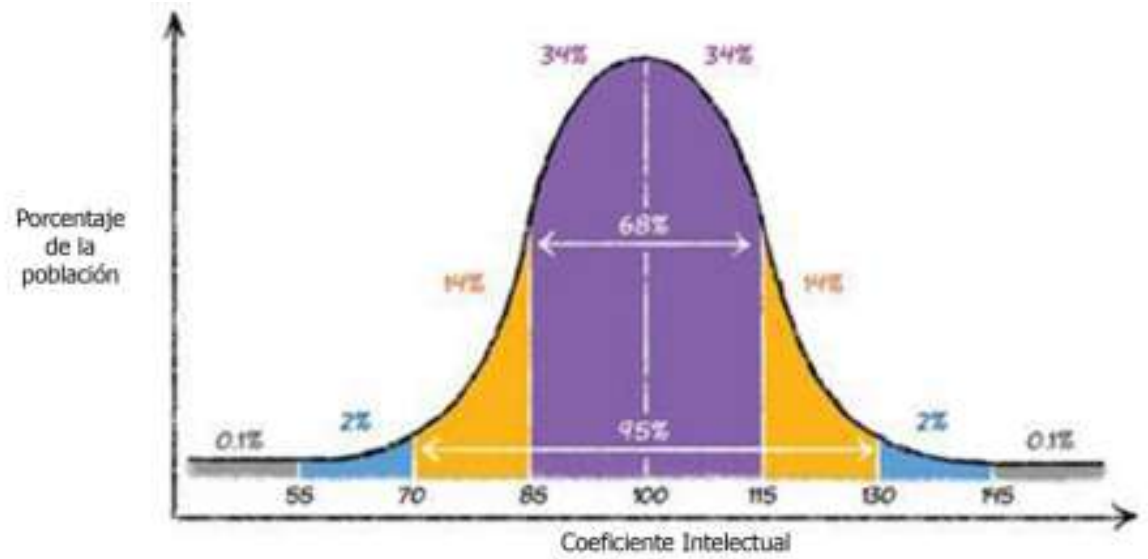

Figura 1. Campana o curva de Gauss para exámenes estandarizados estadunidenses (tomada de Kaufman, 2013)

Para los EXANI, la desviación es de 100 (CENEVAL, 2013b), otro número fácilmente divisible, lo que implica que el 68\% de la población tendrá un CI o índice Ceneval (ICNE) entre 900 y 1100 (ver figura 2).

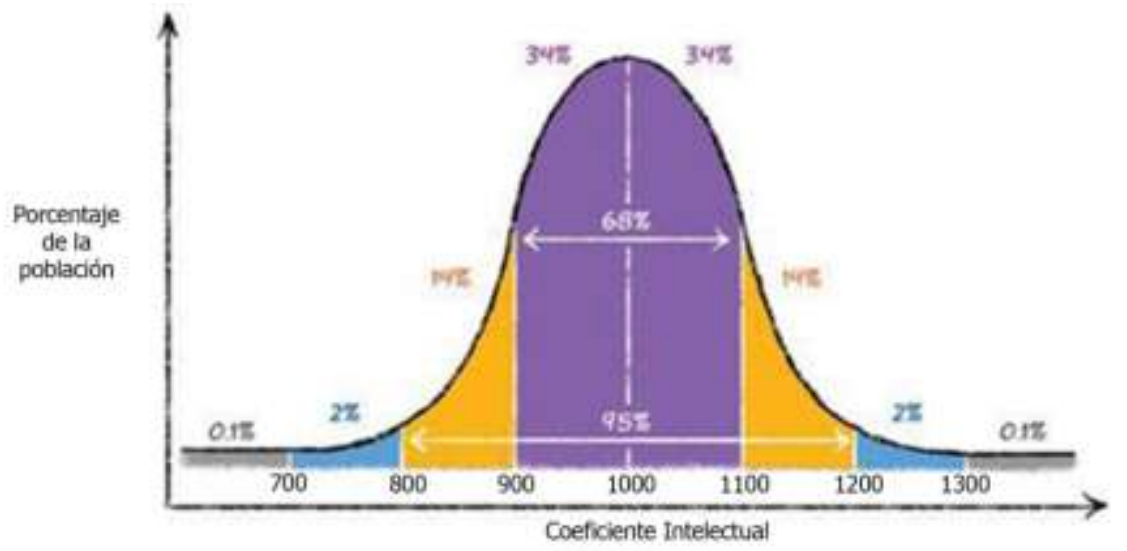

Figura 2. Campana o curva de Gauss para exámenes tipo EXANI y EGEL

Según Montgomery (2013), en los EUA suele considerarse a una persona dotada o talentosa en términos de sus habilidades de procesamiento de información, cuando obtiene un puntaje de 115, o uno que sea mayor o igual a una desviación estándar con respecto al CI promedio o media teórica de la prueba. Aunque el CENEVAL no hace una clasificación para etiquetar a las personas en base al ICNE obtenido en los EXANI, sí lo hace para los exámenes tipo EGEL, donde un ICNE entre 1150 y 1300 se considera sobresaliente (ver figura 3). Consecuentemente, para el caso de los EXANI, sería factible plausible considerar a una persona dotada o sobresaliente si obtiene una calificación mayor o igual a 1100. 


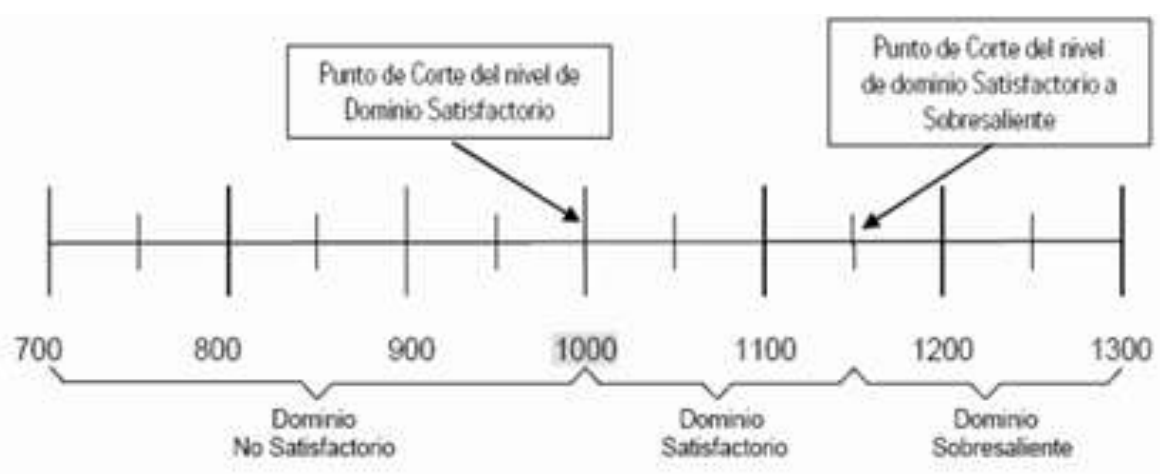

Figura 3. Escala del ICNE para examines tipo EGEL Tomada de (López \& Flores, 2006)

Por último, al igual que los exámenes estadunidenses, los EXANI han ido cambiando a lo largo del tiempo. Por ejemplo, según Martínez, Solís y Osorio (2000), entre 1994 y 1998 el EXANI-II consistía de 180 preguntas, distribuidas de la siguiente forma: Razonamiento Verbal (30); Razonamiento Matemático (30); Mundo Contemporáneo (24); Ciencias Naturales (24); Ciencias Sociales y Humanidades (24); Matemáticas (24); Español (24); a partir de 1999 el CENEVAL dispuso 10 mediciones específicas, dejando la opción de elegir a aquellas que cada IES considerara pertinentes. De manera que para las siete áreas mencionadas se redujeron los reactivos a 120, quedando distribuidos de la siguiente manera: Razonamiento Verbal (20); Razonamiento Matemático (20); Mundo Contemporáneo (16); Ciencias Naturales (16); Ciencias Sociales y Humanidades (16); Matemáticas (16); Español (16). En su versión de 2013, este examen contiene 100 reactivos y las áreas son las siguientes: Razonamiento Lógico-Matemático (20), Razonamiento Verbal (20), Matemáticas (20), Español (20), TIC (20).

Por su parte, el EXANI-III se empezó a utilizar en 1996 y es el que menos cambios ha sufrido. En particular su estructura sigue siendo la misma desde 1996 (CENEVAL, 2007; CENEVAL, 2013b): Razonamiento Lógico-Matemático, Razonamiento Verbal, Metodología y Habilidades para la Investigación, TIC e inglés. Sólo el número de reactivos por área ha cambiado. Para el periodo 1996-2007 es el siguiente: Razonamiento Lógico-Matemático (33), Razonamiento Verbal (33), Metodología y Habilidades para la Investigación (26), TIC (14), Inglés (14). A partir de 2007, este examen está distribuido de la siguiente manera (CENEVAL, 2007; CENEVAL, 2011; CENEVAL, 2013b): Razonamiento Lógico-Matemático (30), Razonamiento Verbal (30), Metodología y Habilidades para la Investigación (30), TIC (20), Inglés (20). 
Habiendo establecido los fundamentos de los exámenes estandarizado de admisión, en los dos siguientes sub-apartados se aborda el análisis de la validez predictiva de los EXANI II y III.

\section{Validez predictiva del EXANI-II}

En el estudio realizado por Martínez et al. (2000) se analizaron los resultados del EXANI-II, de los alumnos aceptados en los años 1996 (121 alumnos), 1997 (127 alumnos), 1998 (148 alumnos) y 1999 (156 alumnos), en la Facultad de Química de la Universidad Autónoma del Estado de México (UAEMEX). En particular, se encontró que existe una correlación promedio de 0.408 , entre el ICNE obtenido por estos estudiantes, y el promedio global que obtuvieron en el primer semestre de la Licenciatura. Las áreas del examen con mayor correlación con el promedio en las asignaturas del primer semestre fueron: a) ciencias naturales (0.280), matemáticas (0.231) y razonamiento verbal (0.202). Sin embargo, también se encontró que si se tomaba el promedio obtenido en el bachillerato, en lugar del ICNE, la correlación promedio cambiaba a 0.568 y, que si además, se incluían las áreas de razonamiento verbal $(\mathrm{RV})$, razonamiento matemático $(\mathrm{RM})$ y matemáticas $(\mathrm{M})$, esta correlación aumentaba hasta 0.616 .

Ponce y García (2003) analizaron a la población de alumnos aceptados que hicieron el EXANI-II en 1999 (i.e., 2757) para ingresar a alguna de las licenciaturas que oferta la UAEMEX en el campus Toluca y que concluyeron el primer semestre con calificación o nota aprobatoria. Los resultados fueron que: el ICNE tuvo una correlación de 0.339 , mientras que el área de RV y de Ciencias Naturales $(\mathrm{CN})$ tuvieron una correlación de 0.495 , seguida por el promedio obtenido en el bachillerato (i.e., 0.421).

Por su parte, Chain, Cruz, Martínez y Jácome (2003) analizaron la información disponible de los resultados de la aplicación del EXANI II y la trayectoria escolar desplegada por el total de estudiantes de primer ingreso (i.e., 6,937) a la Universidad Veracruzana (UV) en 1998. Este estudio, a diferencia de los dos anteriores, siguió la trayectoria de los estudiantes a lo largo de toda la carrera. Esta trayectoria, que fue la variable dependiente, se construyó a partir de tres indicadores básicos: el índice de aprobación en ordinario (IAO), el índice de promoción (IP) y el promedio (PROM). Mediante la aplicación de pruebas de independencia condicional y medidas de correlación simple, encontraron que las variables más relevantes 
asociadas con la trayectoria fueron razonamiento verbal (RV) y español (ESP). Adicionalmente, RV y ESP fueron las que mayor correlación presentaron con el promedio: 0.240 y 0.220 respectivamente. En particular, ninguna otra variable modificó significativamente las probabilidades de los distintos valores de la variable trayectoria.

Cortés y Palomar (2008), estudiando la validez predictiva del proceso de admisión en el rendimiento académico de alumnos de primer año de la Licenciatura en Psicología (N=240), de la Universidad Iberoamericana, campus Ciudad de México, encontraron que el ICNE tuvo una correlación de 0.360. Sin embargo, también encontraron que el promedio de las áreas de conocimiento del EXANI-II (mundo contemporáneo, ciencias naturales, ciencias sociales y humanidades, matemáticas y español) tuvo una correlación ligeramente más alta: 0.371. Siendo el área de ciencias sociales la que individualmente tuvo mayor correlación: 0.304. En el análisis de regresión múltiple, la variable que más porcentaje de varianza predijo con respecto al primer año de licenciatura, fue el promedio de bachillerato (Beta=0.352), seguida por el ICNE (Beta=0.209).

En 2009, Morales, Barrera y Garnnet (2009), estimaron los índices de validez concurrente y predictiva del EXANI-II, de los alumnos aceptados en cualquier facultad o escuela de la UAEMEX durante el periodo 2000-2005. La base del estudio se conformó por una población de 16 mil 756 registros de aspirantes a ingresar a la universidad durante el periodo antes mencionado, que fueron seleccionados mediante el sistema tradicional del EXANI-II. En particular, se pudo corroborar la existencia de una asociación positiva -estadísticamente significativa- entre el promedio general de los alumnos al primer año en la universidad y el ICNE. Sin embargo, la correlación fue relativamente baja: 0.270. El promedio de bachillerato mostró mayor validez predictiva, con un coeficiente de correlación de 0.400. Además, dependiendo de la licenciatura, las áreas de conocimiento del EXANI mostraron coeficientes superiores de correlación que el ICNE, siendo la de Ciencias Naturales la que mayor índice mostró $(r=0.328)$, seguida por Matemáticas $(r=0.270)$.

La literatura sobre la validez predictiva del EXANI-II es escasa. En 19 años la comunidad científica mexicana sólo ha publicado 5 estudios $^{2}$ y, la mayoría de ellos, dentro de la

\footnotetext{
${ }^{2}$ Es posible que existan más estudios. Sin embargo, formarían parte de lo que Rogel, citada por López (2011), denomina literatura "no convencional", "semipublicada" o "fugitiva". Es decir, publicaciones que se escapan de
} 
UAEMEX. Sin embargo, a partir del número de alumnos analizados $(24,485)$ y del periodo de tiempo analizado (1996-2008) se puede concluir que la idoneidad del EXANI-II, como el mejor recurso para decidir la aceptación o rechazo de una persona a estudios de nivel superior, queda en entredicho. El promedio de bachillerato ha resultado, en 3 de los 5 estudios presentados, como mejor predictor, con una correlación promedio de 0.463. Este es un resultado consistente con los presentados por Atkinson y Geiser (2009) que indican que el promedio de bachillerato (i.e., high-school grades) es mejor predictor de éxito en las licenciaturas estadunidenses, que los exámenes estandarizados de admisión como el SAT (Scholastic Aptitude Test) o el ACT (American College Test).

\section{Validez predictiva del EXANI-III}

En un estudio sobre la pertinencia o conveniencia del EXANI-III como requisito para la selección de aspirantes a las maestrías de la Unidad Profesional Interdisciplinaria de Ingeniería y Ciencias Sociales y Administrativas (UPICSA) del Instituto Politécnico Nacional (IPN), Mazcorro, Aday y Hernández (2007), realizaron pruebas de correlación entre el ICNE y las calificaciones de los 5 exámenes que forman la parte sustantiva del proceso de admisión (i.e., Contabilidad, Administración, Economía, Programación Lineal y Probabilidad). Los índices de correlación que encontraron fueron muy bajos: Contabilidad (-0.044), Administración (0.095), Economía (-0.018), Programación Lineal (0.120) y Probabilidad (0.33). También se hizo un análisis de predicciones (AP) y, dado que los resultados de un AP (i.e., el valor de la variable delta) pueden interpretarse como una correlación (Crittenden, Claussen \& Kozlowska, 2007), se encontró que las correlaciones del ICNE con las calificaciones de cada tipo de examen fueron la siguientes: Contabilidad (-0.119), Administración (-0.069), Economía (0.017), Programación Lineal (0.051) y Probabilidad (0.218).

Solís (2009), por su parte, analizó las diferencias entre los estudiantes que se gradúan y los que no obtienen el grado en el posgrado en construcción de la Facultad de Ingeniería de la Universidad Autónoma de Yucatán (UADY). Como parte de este estudio llevó a cabo análisis de regresión, teniendo como variable dependiente al promedio general de calificaciones de la maestría (PGM) y, como variables independientes: promedio general de licenciatura (PGL), promedio de las asignaturas del área de construcción cursadas en el programa de licenciatura (PAC) y calificación general de la prueba EXANI-III (EXA). Los resultados fueron 
significativos para la variable independiente PGL, con un coeficiente de correlación de 0.312. Con las variables PAC y EXA los modelos fueron no significativos (con alfas de 0.46 y 0.88 respectivamente) y valores de $\underline{r}$ de 0.112 y 0.030 . También realizó pruebas de comparación de medias entre los estudiantes que obtuvieron el grado y aquellos que no lo obtuvieron. No se encontraron diferencias significativas para la variable EXA entre ambos grupos.

Sevilla, Martín y Guillermo (2009), a partir de las cohortes que egresaron del año 2005 al 2008 (i.e., 236 alumnos) de tres programas de posgrado (i.e., MIE, MINE y ED) de la Facultad de Educación de la UADY, trataron de identificar información para elaborar un modelo del proceso de selección para cada programa, acorde a sus características, contextos y tendencias. El estudio se centró en las diferencias entre los que lograron obtener el grado de Maestro antes de un año y los que lo hicieron después de un año. Las variables fueron:

$$
\begin{aligned}
& \mathrm{X}_{1}=\text { Puntuación global del EXANI III } \\
& \mathrm{X}_{2}=\text { Puntuación en razonamiento matemático EXANI III } \\
& \mathrm{X}_{3}=\text { Puntuación en razonamiento verbal EXANI III } \\
& \mathrm{X}_{4}=\text { Puntuación de la entrevista } \\
& \mathrm{X}_{5}=\text { Puntuación en inglés EXANI III } \\
& \mathrm{X}_{6}=\text { Promedio de licenciatura }
\end{aligned}
$$

Estadísticamente, se calcularon las medias de los puntajes de cada una de estas variables, tanto para el grupo de los que lograron el grado en tiempo y forma, como para los que no lo lograron. Posteriormente, con el propósito de averiguar si las diferencias observadas entre las medias de ambos grupos eran significativas, se aplicó la prueba $\underline{t}$ para muestras independientes. En particular, para los posgrados MIE y MINE se observó que sólo existen diferencias significativas, entre ambos tipos de graduados, en la puntuación de la entrevista: MIE ( $t=-$ 2.121, $\mathrm{p}<0.05)$, MINE $(t=-3.396, \mathrm{p}<0.05)$. Mientras que para ED fue el promedio de licenciatura: $t=-2.362, \mathrm{p}<0.05$.

La escasez de estudios sobre la validez predictiva del EXANI-III dificulta una posición conclusiva. Mientras que para el EXANI-II se tenía la población completa de alumnos 
aceptados en dos universidades (UAEMEX y UV) y para las licenciaturas ofertadas en el periodo analizado, a 17 años de que empezó a utilizarse el EXANI-III como instrumento para la selección de estudiantes de posgrado, sólo se han hecho tres estudios y a nivel maestría. Sin embargo, la poca evidencia disponible indica que el EXANI-III no tiene utilidad predictiva.

Entonces, ¿qué se puede usar como instrumento fiable para la selección de doctorandos? Ya hemos visto que analizar la historia escolar, en términos de promedios generales, así como la utilización de exámenes de conocimiento, da mejores resultados que los EXANI. En el siguiente apartado se abordarán los aspectos psicológicos más estudiados en los últimos trece años y, que más relación tienen con la función de los estudios de Doctorado.

\section{Factores psicológicos para el éxito en estudios de Doctorado}

La obtención del grado de Doctor, sin importar la disciplina, significa que el graduado es capaz de hacer contribuciones independientes, mediante la investigación original y la erudición, al estado de una cuestión (Lovitts, 2005). La conclusión exitosa de la tesis doctoral marca la transición entre ser un estudiante y el inicio de una carrera como investigador independiente.

Según Lovitts (2005; 2008), durante esta transición los estudiantes deben emigrar de ambientes fuertemente controlados y delimitados, donde su actividad central es consumir el conocimiento parcelado en cursos o seminarios, tareas y lecturas, a uno poco estructurado, donde su tarea será la generación de nuevo conocimiento. En palabras de Azuma (2014), la gente juzga a un doctor recientemente graduado por su investigación, y no por las calificaciones o notas que obtuvo en clase; su éxito está en completar un programa de investigación y construir una línea propia de exploración científica.

Adicionalmente, como subrayan Spaulding y Rockinson (2012), hacer un Doctorado implica asumir riesgos. Un estudiante de doctorado enfrenta tal demanda de esfuerzo y dedicación que su vida personal y social se ve fuertemente acotada, a tal grado que luego se convierte en razón para el abandono. Por ejemplo, varios estudios (Ali \& Kohun, 2006; Gardner, 2009; Lovitts, 2005 ) indican que en los EUA, al menos un $40 \%$ de los estudiantes que se ma- 
triculan en un programa de doctorado abandonan los estudios antes de obtener su candidatura y, entre quienes la obtienen, alrededor del $25 \%$ terminan rindiéndose. Una situación similar ocurre en Australia (Jiranek, 2010), donde alrededor del 40\% de los doctorandos, dependiendo del área de conocimiento, abandonan sus estudios doctorales.

La literatura registra varios modelos teóricos (Jiranek, 2010; Lovitt, 2005; Smith et al., 2006; Spaulding \& Rockinson, 2012; Wao \& Onwuegbuzie, 2011) para identificar y analizar los factores que hacen que un estudiante de doctorado se convierta en productor, más que consumidor de conocimiento. Entre ellos se perciben diferencias, pero la coincidencia más significativa es que todos muestran que la obtención del grado de doctor, es un proceso longitudinal donde los factores personales condicionan la efectividad de las estrategias institucionales (ver figuras 4 y 5 ).

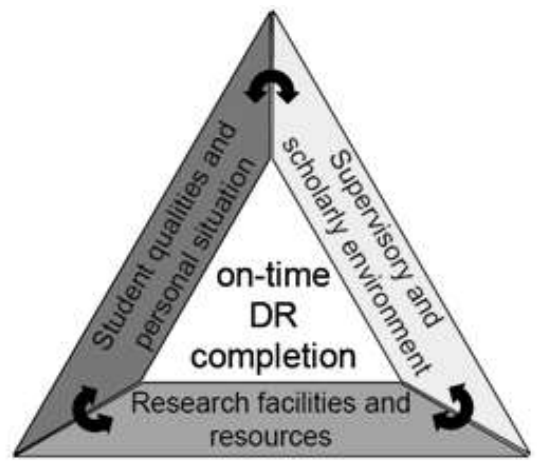

Figura 4. Modelo de Jiranek (2010): factores que afectan el éxito en el doctorado

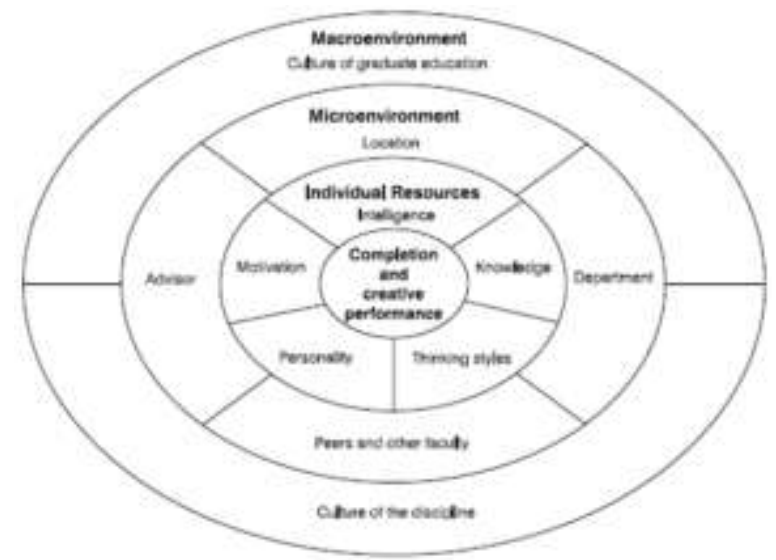

Figura 5. Modelo de Lovitts (2005): factores que afectan el éxito en el doctorado 
Algunos estudios (Ahern \& Manathunga, 2004; Kearns, Gardiner, \& Marshall, 2008) señalan que los doctorandos que desertan presentan comportamientos de auto-sabotaje o autoobstaculización. Su desempeño está marcado por actitudes de procastinación, perfeccionismo y sobrecompromiso con actividades distintas a las de su programa doctoral. Otros estudios, como el de Ali y Kohun (2006), indican que las emociones y los sentimientos de aislamiento están entre los factores que más inciden en la deserción en estudios de Doctorado. Según este estudio, esos sentimientos derivan de una insuficiente comunicación entre los estudiantes y, de estos, con la planta académica o docente.

En particular, aquellos estudiantes que logran evitar estas conductas y obtener el grado de Doctor, en los tiempos establecidos, regularmente son más persistentes y focalizados en su tarea (Kearns et al., 2008). Sus deseos por alcanzar la cima del éxito académico (Brailsford, 2010) se acompañan de disposición para atender y superar los desafíos y sacrificios asociados con los estudios de doctorado (Spaulding \& Rockinson, 2012). Es decir, tienen razones intrínsecas (e.g., interés en su tema de investigación) y se comprometen más decididamente con la consecución de sus estudios (Ahern \& Manathunga, 2004). En la siguiente sección se analizan algunos factores psicológicos específicos (i.e., un subconjunto de los factores personales) que la literatura registra como los de mayor significado en la ruta de obtener el grado de Doctor, con éxito. Se exponen, buscando ubicarlos como complemento, si no es que como alternativa, a los recursos tradicionales con que se procesa la admisión a los programas educativos (e.g. EXANI) y que en las secciones previas se han reconocido como insuficientes.

\section{Valor}

Este factor es considerado por Duckworth, Peterson, Matthews, y Kelly (2007) como uno de los recursos psicológicos más compartidos entre la élite científica (valor ${ }^{3}$, traducido del inglés "grit"). El valor es un subcomponente de uno de los cinco grandes aspectos de la personalidad, denominada consciencia o minuciosidad (Almlund, Duckworth, Heckman, \& Kautz, 2011). Duckworth et al. (2007) lo definen como perseverancia y pasión para lograr metas a largo plazo. Esto implica trabajar con energía hacia desafíos, mantener el esfuerzo y el interés durante años a pesar del fracaso, la adversidad y períodos de poco o ningún progre-

\footnotetext{
${ }^{3}$ Escogimos valor sobre perseverancia debido a que la definición del primer término es mucho más coincidente con la definición de "grit" que la del segundo: Cualidad del ánimo, que mueve a acometer resueltamente grandes empresas y a arrostrar los peligros.
} 
so en los objetivos establecidos. El individuo valiente emprende sus metas y objetivos como un maratón. Su ventaja sobre los demás no es su inteligencia, sino su aguante, persistencia y resistencia. Mientras que la decepción o el aburrimiento señalan a otros que es hora de cambiar de trayectoria y disminuir las pérdidas, el individuo valiente mantiene el curso. Por tanto, hay que diferenciar el valor, de su constructo hermano el auto-control, también llamado autodisciplina o auto-regulación, pues los individuos auto-disciplinados, pero con valor moderado o bajo, tienden a ser poco constantes en sus metas e intereses a lo largo del tiempo.

En un análisis de este subcomponente de la personalidad, Duckworth et al. (2007) realizaron seis estudios donde se evaluó el rol del valor. En los dos primeros estudios, $(N=1545)$ y $(N=690)$, encontraron que los adultos mayores de 25 años con más valor alcanzaron niveles educativos más altos; así como, significativos niveles de correlación entre éste y el nivel educativo. En el tercer estudio ( $N=138)$, con estudiantes de Licenciatura, se encontró que las personas con más valor obtuvieron promedios más altos en sus calificaciones e incluso mayores que los de aquellas que tenían un CI más alto. (i.e. $r=0.250$ ). En un informe subsecuente de otros cinco estudios, Duckworth y Quinn (2009) encontraron que el valor estuvo inversamente relacionado con el número de cambios de carrera u ocupación. De igual forma, encontraron una correlación significativa (i.e., 0.298) entre el valor y el promedio final del año, de estudiantes de secundaria y primero de bachillerato, todos con un alto nivel de éxito.

En un estudio más reciente con 140 estudiantes masculinos afroamericanos de licenciatura, matriculados en una universidad pública estadunidense, predominantemente blanca, Strayhorn (2014) encontró que el valor predijo el $24 \%$ de la varianza en el promedio de estos estudiantes; con una correlación de 0.490. En conjunto, esta evidencia da elementos para afirmar que el valor resulta una variable de interés predictivo para el éxito a lo largo de diferentes niveles educativos.

\section{Autodisciplina y autorregulación}

La capacidad de las personas para regular sus pensamientos, sus creencias y sus acciones en pro de un propósito, suele identificarse con los nombres de autodisciplina, autoregulación, o autocontrol (Duckworth \& Carlson, 2013; Gong, Rai, Beck, \& Heffernan, 2009; Muammar, 2011; Oaten \& Cheng, 2006). Aunque entre estos términos pueden distinguirse diferencias relacionadas con niveles de conciencia práctica, en general hacen referencia a una condición psicológica, desde la que la persona intenta dominar los recursos, la actitud y la 
ruta que le permite lograr su propósito. La autoregulación es una especie de disposición personal para perseguir y alcanzar la meta deseada, que condiciona todo esfuerzo, estrategia y estados de ánimo que se le asocien (e.g. Pasternak, 2013).

Ejemplos de autocontrol son el modular la ira en lugar de hacer una rabieta, leer las instrucciones de la prueba antes de pasar a las preguntas, prestar atención a un profesor en lugar de soñar despierto, elegir hacer la tarea o los deberes en lugar de ver televisión, dedicar más tiempo a los estudios para incrementar los aprendizajes, así como persistir en tareas a largo plazo, a pesar del aburrimiento y la frustración.

Sin embargo, de la Fuente y Justicia (2007) proponen que, al hablar de los procesos de regulación en ambientes académicos, es necesario flexibilizar los alcances de los conceptos de aprendizaje y enseñanza. Desde su perspectiva, la complejidad de estos conceptos obliga a reconocer los procesos educativos con mayor apertura y aceptar que hay condicionantes derivadas tanto del aprendiz, como de la enseñanza y del contexto en el que se actúa. La autoregulación es un producto con influencia directa de condicionantes personales de quien aprende, tanto como de las acciones de enseñanza, de la tarea y del ambiente en que se lleva a cabo. Un estudiante no es insensible a la propuesta de enseñanza que recibe, a la naturaleza del contenido que enfrenta, ni al ambiente en el que se presenta la tarea. Por ello, entender la autoregulación como un producto de la persona es limitarla, al mismo tiempo que dejarle la responsabiliad absoluta de su ocurrencia.

En dos estudios realizados entre estudiantes de secundaria ( $N=140$ y $N=164)$, Duckworth y Seligman (2005) informan que los adolescentes con un alto nivel de autocontrol, superaron a sus pares más impulsivos en cada variable de rendimiento académico, incluyendo la asistencia a clase, las calificaciones o notas en sus respectivas boletas o libretas, los resultados de pruebas estandarizadas de rendimiento y la admisión a una escuela competitiva de estudios de bachillerato. En particular, en el segundo estudio encontraron una alta correlación entre la autodisciplina y el promedio final de calificaciones $(r=0.670)$, mientras que la correlación entre el CI y el promedio final fue menor $(r=0.320)$. Adicionalmente, cuando el CI y la autorregulación se introdujeron simultáneamente en un análisis de regresión múltiple, la autodisciplina representó un porcentaje más alto de la varianza del promedio final $(\beta=0.65, p$ $<0.001)$ que el CI $(\beta=0.25, p<0,001)$. También fue un predictor del número de horas dedicadas a realizar tareas o deberes: $r=0.35, p<0.001$. 
En su artículo sobre género y autocontrol, Duckworth y Seligman (2006) presentan los resultados de dos estudios. En el primero, con 140 estudiantes de octavo grado de una escuela del noreste de EUA, al comparar las puntuaciones de autodisciplina, las niñas tuvieron mejores resultados que los niños, $t(138)=4,12, p=0.001, d=0.71$. Esta variable correlacionó significativamente con el promedio general $(r=0.57, P<0.001)$ y de manera menos robusta con los puntajes en las pruebas estandarizadas de rendimiento $(r=0.29, p=0.001)$. Adicionalmente, se encontró que la autodisciplina pronosticó el promedio general cuando se controló sexo $(\beta=0.50, \mathrm{r}=0.47, p=0.001)$. Posteriormente, Duckworth, Tsukayama y May (2010), en un estudio con alumnos de quinto y octavo grado, encontraron que la autorregulación impacta positivamente el rendimiento académico, y que los cambios en la autodisciplina a través del tiempo predicen los cambios en el promedio general de los estudiantes: $\beta_{30}=1.81, t(610)=$ 4.47, $p<.001)$. Así, autodisciplina parece ser un factor de importancia para el desempeño académico exitoso.

\section{Propósito o meta}

El diccionario de la Real Academia Española caracteriza al término Propósito como polisémico, donde sus dos primeras acepciones son: a) Ánimo o intención de hacer o de no hacer algo y b) Objetivo que se pretende conseguir. La definición en la psicología anglosajona es una fusión de las dos acepciones anteriores y se dicute desde construcciones teóricas que igualmente refieren a las metas o a los logros, como equivalentes (e.g. de la Fuente, 2004; Was, 2006;). Hulleman, Schrager, Bodmanny Harackiewicz (2010) definen el propósito como: "una representación cognitiva enfocada en el futuro, que orienta el comportamiento hacia un estado final, relacionado con la competencia, que el individuo se ha comprometido a abordar/afrontar o a evitar/rehuir"4.

De acuerdo con Senko, Hulleman y Harackiewicz (2011) existen cuatro tipos de propósitos: 1) propósitos enfocados al rendimiento (performance-approach), es decir, propósitos en los que la persona trata de superar a los demás, o trata de parecer talentosa; 2) propósitos de evasión del rendimiento (performance-avoidance), en los que el individuo intenta que su rendimiento no esté por debajo de otros, o evita aparecer como menos talentoso; 3) propó-

\footnotetext{
${ }^{4}$ Esta es una traducción libre de los autores. El original en inglés reza: “a future-focused cognitive representation that guides behavior to a competence-related end state that the individual is committed to either approach or avoid."
} 
sitos orientados al dominio o maestría (mastery-approach), donde la persona busca aprender o mejorar sus habilidades y 4) propósitos centrados en rehuir el dominio (mastery-avoidance), en los que el individuo pretende evitar el fracaso en el aprendizaje o la perdida de habilidad. En este apartado se tratan únicamente los propósitos 1 y 3.

Hulleman et al. (2010) realizaron un meta-análisis de 243 estudios en los que participaron un total de 91,087 personas. Estos estudios utilizaron diferentes escalas para medir el Propósito y, abarcaron desde el nivel básico de educación hasta el de Licenciatura. En particular, los propósitos orientados al rendimiento tuvieron una correlación con los resultados de desempeño o rendimiento (i.e., el éxito académico) de $r \wedge=0.14$. Mientras que los orientados a la maestría tuvieron un valor de $r^{\wedge}=0.05, t(64)=2.64, p<.05$. Sin embargo, al medir la correlación de éste último propósito con el interés, la motivación intrínseca y la curiosidad, la correlación cambio a $r^{\wedge}=0.44, t(51)=15.98, p<.01$. Una revisión de 24 estudios hecha por Senko, Hulleman y Harackiewicz (2011) valida estos resultados. En particular, la evidencia acumulada muestra que los propósitos orientados al rendimiento, a menudo se asocian con altas calificaciones, independientemente del nivel de confianza y la estrategia de aprendizaje (i.e., profunda o superficial) del estudiante. Sin embargo, en contextos que requirieron una comprensión profunda del material de la clase, así como la aplicación, análisis y síntesis de los conceptos del curso, los resultados fueron mixtos entre los propósitos de rendimiento y maestría.

Algunos estudios se han centrado en las emociones (i.e., diversión, aburrimiento, enojo, esperanza, orgullo, ansiedad, desesperación y vergüenza) que produce el seguir uno u otro propósito, mientras que otros han puesto atención a la relación entre el propósito y la forma en que el conocimiento se transfiere y prepara a los estudiantes para aprender en el contexto de nuevas oportunidades educativas. En un estudio del primer tipo con 218 estudiantes universitarios (147 mujeres y 71 varones) en un curso de psicología que tomaron parte en el estudio a cambio de créditos adicionales (edad: $M=19.43, S D=1.76$ años ), Pekrun, Elliot y Maier (2009) encontraron que la esperanza y el orgullo eran las emociones que mejor predijeron el rendimiento en términos de calificación o nota en en un examen (i.e., $F(1,213)=$ $14.42, p<.001(\beta=0.27)$ y $F(1,213)=17.34, p<.001(\beta=0.29)$ respectivamente). Mientras que el propósito orientado al rentidimiento fue el predictor más fuerte para el rendiemiento en este mismo examen: $F(1,214)=14.15, p<.001(\beta=0.38)$. Aunque la relación entre las metas de dominio y el desempeño no fue significativa (i.e., $F(1,214)=3.02, p=0.08(\beta=$ $0.11)$ ), este tipo de metas fueron un factor predictivo negativo del aburrimiento (i.e., $F$ (1, 
$214 ..)=50.54, p<.001(\beta=-.43)$ Esto es importante porque el aburrimiento fue un predictor negativo casi significativo con respecto al rendimiento: $F(1,213)=3.71, p=0.055(\beta=-$ $.14)$.

Por su parte, Belenky y Nokes-Malach (2012), en un estudio con 104 estudiantes de psicología de la Universidad de Pittsburg, encontraron que el propósito orientado a la maestría puede servir como un mecanismo de transferencia para facilitar los procesos cognitivos constructivos y ayuda a conectar episodios posteriores de aprendizaje, con el aprendizaje anterior relevante $(\beta=0.37)$. Este resultado, así como la relación del propósito de dominio o maestría con el interés, la motivación intrínseca, la curiosidad, la comprensión profunda de un tema y, la aplicación, análisis y síntesis de conceptos, hacen que este constructo sea de particular interés para oportunidades educativas que requieran este tipo de habilidades.

\section{Creatividad}

De los constructos analizados en este artículo, el denominado creatividad quizá sea el más elusivo. Por ejemplo, Plucker, Beghetto y Dow (2004), encontraron 34 definiciones diferentes para este constructo, cada una abordando un matiz diferente. Por tal razón es fácil encontrar diferentes formas de evaluar el potencial creativo de las personas (Kaufman, Plucker $\&$ Russell, 2012). En este artículo se adopta la definición de Eco (2007, p.78) por ser lo suficientemente general y rigurosa como para incluir a las definiciones anteriores, así como las nuevas concepciones de creatividad señaladas por Kaufman et al. (2009).

Para Eco (2007), la creatividad es aquella actividad que produce algo inédito que la comunidad estará dispuesta a reconocer, a aceptar, a hacer propio y a reelaborar, en el largo plazo, y que se convierte en patrimonio colectivo, a disposición de todos, y no para disfrute personal exclusivo. Así mismo, para que la creatividad sea digna de tal nombre ha de estar imbuida de actividad crítica.

No es creativa la idea surgida en el transcurso de un brainstorming (lluvia de ideas), lanzada tan sólo por probarlo todo, y aceptada entusiásticamente a falta de algo mejor. Para que la idea sea creativa, ha de ser analizada y, al menos en el caso de la creatividad científica, ha de ser susceptible de falseamiento. Consecuentemente, argumenta Eco (2007), la creatividad se desarrolla por innovación, es decir mediante la invención de ideas nuevas, pero tam- 
bién mediante la crítica del saber o de las prácticas anteriores, y sobre todo a través de la crítica del propio discurso.

La creatividad, al incluir acciones innovadoras, con frecuencia se confunde con CI. Para analizar la relación entre CI y potencial creativo, representado este último por el razonamiento divergente (divergent thinking), es decir, por la habilidad para generar muchas respuestas a problemas abiertos y multifacéticos (Gibson, Folley, \& Park, 2009), Kim (2005) revisó 21 estudios con un total de 45,880 participantes. Lo que encontró fue que la correlación media entre $\mathrm{CI}$ y razonamiento divergente fue pequeña: $r=.174 ; 95 \%$ IC $=.165-.183$. Y que cuando los coeficientes intelectuales de los participantes se dividieron en 4 niveles, las correlaciones entre CI y potencial creativo fueron las siguientes: $C I<100[r=.260]$; $100<C I\rangle$ $120[r=.140] ; 120<I Q>135[r=.259] ; I Q>135[r=-.215])$.

En una revisión subsecuente de 44 estudios diferentes, donde participaron 52,741 personas, se logró conocer los coeficientes de correlación entre el CI, la calificación en las pruebas de razonamiento divergente (RD) y el Logro Creativo (LC) que no es más que la suma de los productos creativos generados por un individuo en el curso de su vida (Carson, Peterson, \& Higgins, 2005). Kim (2008) también exploró la correlación promedio entre el CI y el LC y encontró que fue de 0.167 (95\% IC=0.141 - 0.193). Mientras que la correlación promedio entre los resultados de las pruebas de RD y el LC fue de 0.216 (95\% IC $=0.207-0.225$ ). Adicionalmente, los coeficientes de correlación entre las puntuaciones medias de dichas pruebas y el LC en el arte, la escritura, la ciencia, y las habilidades sociales, fueron de $r=0.232, r=$ 0.187, $r=0.166$ y $r=0.171$ respectivamente. Mientras que los coeficientes de correlación promedio entre el CI y el LC, para las mismas áreas, fueron de $r=0.056, r=0.172, r=0.061$ y $r=0.119$. Las sub-escalas de elaboración y fortalezas (Kim, 2006) fueron las de mayor correlación con el logro creativo: 0.300 y 0.322 respetivamente.

En síntesis, los valores que ofrece la evaluación de creatividad y su relación con el LC parecen ser indicadores que abonan a la identificación de variables para el éxito educativo, así como del éxito en otras áreas. No deja de llamar la atención, sin embargo, que la correlación entre RD y LC en la ciencia $(r=0.166)$ sea casi la misma que la que se detectó entre CI y LC en ciencia $(r=0.167)$. 


\section{Discusión y conclusiones}

La formación universitaria, particularmente la de postgrado, busca tener niveles crecientes de eficiencia manifiestos en el número de graduados y el nivel de producción científica que logren. Para ello se han sofisticado los procesos de selección de aspirantes con exámenes estandarizados y evaluaciones que buscan predecir el éxito de los aspirantes en los estudios de posgrado. En esa ruta, muchas pruebas psicológicas y de conocimiento han sido desarrolladas, pero no todas han sido lo eficientes que se esperaban. En este artículo se analizó esta problemática y se mostró evidencia de que la aplicación de pruebas estandarizadas no ofrece la certidumbre deseada para tales propósitos. En particular se ofreció evidencia de que se puede prescindir del EXANI-II y utilizar el promedio de bachillerato y los exámenes de conocimiento por áreas particulares (e.g., Ciencias Naturales y Matemáticas), como instrumentos de selección y predicción del éxito académico en Licenciatura (e.g., Morales, Barrera, \& Garnnet , 2009). No obstante, estudios que cubran la variedad de tipos de bachillerato (e.g., tecnológico y propedéutico) y que incluyan más cohortes y más universidades (e.g. públicas vs. privadas) son necesarios. Quedan pendientes para estos casos, preguntas como las siguientes: ¿Qué tipo de bachillerato predice mejor el éxito académico? ¿Hay relación entre el tipo de bachillerato estudiado y los resultados que se obtienen en el ICNE?

La utilidad predictiva de la aplicación del EXANI-III para estudios de postgrado tampoco fue alentadora. A pesar de que los estudios que lo documentan son pocos y de baja variedad, el promedio de $\underline{r}$ para las correlaciones simples entre el ICNE y los exámenes de conocimientos aplicados (e.g., Mazcorro, Aday, \& Hernández, 2007) fue bajo en el análisis de predicción (e.g., de 0.059 a 0.020). Adicionalmente, el EXANI-III no tuvo validez predictiva aceptable ( $r=0.030)$ para el promedio general de Maestría en el estudio de Solís (2009). Tampoco fue un elemento predictor, en el mismo estudio, de la obtención del grado de Maestría, en los tiempos establecidos por el programa de posgrado.

Estos datos indican que hay que tener reservas a la hora de utilizar los exámenes estandarizados como recurso eficiente de predicción de éxito académico en posgrado. Se necesitan muchos más estudios para llegar a conclusiones más sólidas. En particular, porque el EXANI-III es ampliamente utilizado por las IES mexicanas para justificar la aceptación o rechazo a estudios de posgrado y porque el CONACYT lo exige como requisito para los programas que están en su padrón de calidad. 
Razón aparte de la imprecisión predictiva de los exámenes estandarizados, la literatura revisada ofrece evidencia de que la selección de aspirantes puede ser más eficiente si incorpora la evaluación de aspectos personales y de trayectoria académica. En cuanto a los constructos psicológicos, se pudo ver que por definición las personas con un alto grado de valor, autodisciplina y propósito, evitan con más éxito las conductas de auto-sabotaje y, a través de la creatividad previenen las emociones, o sentimientos de aislamiento que a la postre refuerzan los impedimentos para el logro de las metas académicas.

A esta mezcla se le pueden añadir más elementos. Sternberg et al. (2012) argumentan que en la selección de estudiantes, también se deben tomar en cuenta aspectos éticos y de valores, y proponen medir el nivel de sabiduría de los postulantes mediante autoinformes. Sin embargo, es necesario subrayar que ninguno de los constructos analizados en este artículo se ha estudiado en postulantes a estudios de posgrado. Por tanto, al menos para el caso de México, es necesario realizar estudios que tomen en cuenta estos elementos y observar los resultados, tal como se está haciendo en los EUA con constructos diferentes a los analizados aquí (Atkinson \& Geiser, 2009; Megginson, 2009; Sternberg et al. 2012).

Por último, además de las limitaciones antes señaladas, en este artículo no se abordaron: a) las implicaciones socio-políticas y administrativas (e.g., Márquez, 2012), más que académicas, que median el uso de los exámenes estandarizados en México y b) los sistemas internos de las IES mexicanas para aplicar evaluaciones fiables. El análisis y discusión de estos y otros aspectos es demasiado amplio y rebasa los límites de esta publicación. Por tanto, constituye parte del trabajo futuro que se debe hacer. 


\section{Referencias}

Ahern, K. y Manathunga, C. (2004). Clutch-starting stalled research students. Innovative Higher Education, 28(4), 237-254. doi:10.1023/B:IHIE.0000018908.36113.a5

Ali, A. y Kohun, F. (2006). Dealing with isolation feelings in IS doctoral programs. International Journal of Doctoral Studies, 1(1), 21-33. Recuperado de http://www.informingscience.us/icarus/journals/ijds/

Almlund, M., Duckworth, A. L., Heckman, J., \& Kautz, T. (2011). Personality Psychology and Economics. Handbook of the economics of education, 4(1). doi: 10.3386/w16822

Atkinson, R. C., y Geiser, S. (2009). Reflections on a century of college admissions tests. Educational Researcher, 38 (9), 665-676. doi: 10.3102/0013189X09351981

Azuma, R. T. (2014). So long, and thanks for the Ph.D.! [Página web] Recuperado el 7 de enero de 2014 de http://www.cs.unc.edu/ azuma/hitch4.html

Belenky, D. M., y Nokes-Malach, T. J. (2012). Motivation and transfer: The role of masteryapproach goals in preparation for future learning. Journal of the Learning Sciences, 21(3), 399-432. doi: 10.1080/10508406.2011.651232

Boake, C. (2002). From the Binet-Simon to the Wechsler-Bellevue: Tracing the history of intelligence testing. Journal of Clinical and Experimental Neuropsychology, 24(3), 383-405. doi: 10.1076/jcen.24.3.383.981

Brailsford, I. (2010). Motives and aspirations for doctoral study: Career, personal, and interpersonal factors in the decision to embark on a history Ph.D. International Journal of Doctoral Studies, $\quad 5, \quad 15-27 . \quad$ Recuperado de http://www.informingscience.us/icarus/journals/ijds/

Busato, V. V., Prins, F. J., Elshout, J. J., y Hamaker, C. (2000). Intellectual ability, learning style, personality, achievement motivation and academic success of psychology students in higher education. Personality and Individual differences, 29(6), 1057-1068. doi: 10.1016/S0191-8869(99)00253-6

Carson, S. H., Peterson, J. B., y Higgins, D. M. (2005). Reliability, validity, and factor structure of the creative achievement questionnaire. Creativity Research Journal, 17(1), 37-50. doi: 10.1207/s15326934crj1701_4

CENEVAL. (2007). Guía del examen nacional de ingreso al posgrado, EXANI III. Centro Nacional de Evaluación para la Educación Superior, A.C. Recuperado de http://www.ceneval.edu.mx 
CENEVAL. (2011). Guía del examen nacional de ingreso al posgrado, EXANI-III. Centro Nacional de Evaluación para la Educación Superior, A.C. Recuperado de http://www.ceneval.edu.mx

CENEVAL. (2013a). Guía del examen nacional de ingreso a la educación superior, EXANIII. Centro Nacional de Evaluación para la Educación Superior, A.C. Recuperado de http://www.ceneval.edu.mx

CENEVAL. (2013b). Guía del examen nacional de ingreso al posgrado, EXANI-III. Centro Nacional de Evaluación para la Educación Superior, A.C. Recuperado de http://www.ceneval.edu.mx

Chain, B., Cruz, N., Martínez, M. y Jácome, N. (2003). Examen de selección y probabilidad de éxito escolar en estudios superiores: Estudio en una universidad pública estatal mexicana. Revista Electrónica de Investigación Educativa, 5(1), 5. Recuperado de http://redie.ens.uabc.mx/index.php/redie/index

Chamorro-Premuzic, T., y Furnham, A. (2003). Personality predicts academic performance: Evidence from two longitudinal university samples. Journal of Research in Personality, 37(4), 319-338. doi: 10.1016/S0092-6566(02)00578-0

Colom, R., Rebollo, I., Palacios, A., Juan-Espinosa, M., y Kyllonen, P. C. (2004). Working memory is (almost) perfectly predicted by g. Intelligence, 32(3), 277-296. doi: 10.1016/j.intell.2003.12.002

Colonia-Duque, S. M. (2010). Caracterización del perfil de los estudiantes de posgrado a partir de la información de admisión y el desempeño académico. Aplicación a la maestría en ingeniería administrativa de la Universidad Nacional Sede Medellín. (Tesis de Maestría).

Recuperada de http://www.bdigital.unal.edu.co/3601/1/42144324.2010.pdf

CONACYT. (2013). Programa Nacional de Posgrados de Calidad: Anexo A. Consejo Nacional de Ciencia y Tecnología. Recuperado de http://www.conacyt.mx/index.php/elconacyt/convocatorias-y-resultados-conacyt/convocatorias-pnpc/resultados-pnpc/919-15/file

Cortés, A., y Palomar, J. (2008). El proceso de admisión como predictor del rendimiento académico en la educación superior. Universitas Psychologica, 7(1), 199-215. Recuperado de http://pepsic.bvsalud.org/scielo.php?script=sci_serial\&pid=1657$9267 \& \operatorname{lng}=$ pt\&nrm $=$ iso 
Crittenden, P. M., Claussen, A. H., y Kozlowska, K. (2007). Choosing a valid assessment of attachment for clinical use: A comparative study. Australian and New Zealand Journal of Family Therapy, 28(2), 78-87. doi: 10.1375/anft.28.2.78

de la Fuente, J. (2004). Perspectivas recientes en el estudio de la motivación: La Teoría de la Orientación de Meta. Electronic Journal of Reserach in Educational Psychology, 2(1), 35-62. Recuperado de http://www.investigacionpsicopedagogica.org/revista/new/index.php

de la Fuente, J. y Justicia, F. (2007). El Modelo DIDEPRO® de Regulación de la Enseñanza y del Aprendizaje: avances recientes. Electronic Journal of Research in Educational Psychology and Psychopedagogy, 13(3), 535-564. Recuperado de http://www.investigacion-psicopedagogica.org/revista/new/index.php

de los Santos, E. (2004). Los procesos de permanencia y abandono escolar en la educación superior. Revista Iberoamericana de Educación, 3(12), 1-7. Recuperado de http://www.oei.es/index.php

Deary, I. J., Penke, L., y Johnson, W. (2010). The neuroscience of human intelligence differences. Nature Reviews Neuroscience, 11(3), 201-211. doi: 10.1038/nrn2793

Duckworth, A. L., Peterson, C., Matthews, M. D., y Kelly, D. R. (2007). Grit: perseverance and passion for long-term goals. Journal of personality and social psychology, 92(6), 1087. doi: 10.1037/0022-3514.92.6.1087

Duckworth, A. L., Quinn, P. D., y Tsukayama, E. (2012). What No Child Left Behind leaves behind: The roles of IQ and self-control in predicting standardized achievement test scores and report card grades. Journal of Educational Psychology, 104(2), 439. doi: $10.1037 / \mathrm{a} 0026280$

Duckworth, A. L., Tsukayama, E., y May, H. (2010). Establishing causality using longitudinal hierarchical linear modeling: An illustration predicting achievement from self-control. Social psychological and personality science, 1(4), 311-317. doi: $10.1177 / 1948550609359707$

Duckworth, A. L., y Quinn, P. D. (2009). Development and validation of the Short Grit Scale (GRIT-S). Journal of personality assessment, 91(2), 166-174. doi: $10.1080 / 00223890802634290$

Duckworth, A. L., y Carlson, S. M. (2013). Self-regulation and school success. En B.W. Sokol, F. M.E. Grouzet, y U. Müller (Eds.), Self-regulation and autonomy: Social and 
developmental dimensions of human conduct. New York, NY: Cambridge University Press.

Duckworth, A. L., y Seligman, M. E. (2005). Self-discipline outdoes IQ in predicting academic performance of adolescents. Psychological science, 16(12), 939-944. doi: 10.1111/j.1467-9280.2005.01641.x

Duckworth, A. L., y Seligman, M. E. (2006). Self-discipline gives girls the edge: Gender in self-discipline, grades, and achievement test scores. Journal of educational psychology, 98(1), 198. doi: 10.1037/0022-0663.98.1.198

Eco, U. (2007). Turning Back the Clock: Hot Wars and Media Populism. Harcourt; First Edition edition.

Esquivel, L. y Rojas, C. (2005). Motivos de estudiantes de nuevo ingreso para estudiar un posgrado en educación. Revista Iberoamericana de Educación Superior, 36(5), 2005. Recuperado de http://www.rieoei.org/index.php

Gagne, R. M., Wager, W. W., Golas, K. C., Keller, J. M. and Russell, J. D. (2005). Principles of instructional design, 5th edition. Performance Improvement, 44: 44-46. doi: 10.1002/pfi.4140440211

Gardner, S. K. (2009). Student and faculty attributions of attrition in high and low-completing doctoral programs in the United States. Higher Education, 58(1), 97-112. doi: $10.1007 / \mathrm{s} 10734-008-9184-7$

Gibson, C., Folley, B. S., y Park, S. (2009). Enhanced divergent thinking and creativity in musicians: A behavioral and near-infrared spectroscopy study. Brain and Cognition, 69(1), 162-169. doi: 10.1016/j.bandc.2008.07.009

Gong, Y., Rai, D., Beck, J. B. \& Heffernan, T. N. (2009). Does Self-Discipline impact students' knowledge and learning? En actas de International Conference on Educational Data Mining (pp. 61-70). Recuperado de http://eric.ed.gov/?id=ED539087

Gray, J. R., y Thompson, P. M. (2004). Neurobiology of intelligence: science and ethics. Nature Reviews Neuroscience, 5(6), 471-482. doi:10.1038/nrn1405

Greeno, J., Collins, A. y Resnick, L. (1996). Cognition and Learning. En Berliner, D. y Calfee, R. (eds.), Handbook of Educational Psychology, Macmillan, New York: pp. $15-46$.

Hernández, A. S. (2007). La Equidad en la Distribución de Oportunidades Educativas en México: Un estudio con base en los datos del EXANI-I. Revista Electrónica Iberoa- 
mericana sobre Calidad, Eficacia y Cambio en Educación, 5(1), 2. Recuperado de http://www.rinace.net/reicenumeros.htm

Hulleman, C. S., Schrager, S. M., Bodmann, S. M., y Harackiewicz, J. M. (2010). A metaanalytic review of achievement goal measures: Different labels for the same constructs or different constructs with similar labels? Psychological bulletin, 136(3), 422. doi: $10.1037 / \mathrm{a} 0018947$

Jaeggi, S. M., Buschkuehl, M., Jonides, J., \& Perrig, W. J. (2008). Improving fluid intelligence with training on working memory. Proceedings of the National Academy of Sciences, 105(19), 6829-6833. doi: 10.1073/pnas.0801268105

Jiranek, V. (2010). Potential predictors of timely completion among dissertation research students at an Australian Faculty of Sciences. International Journal of Doctoral Studies, 5, 1-13. Recuperado de http://www.informingscience.us/icarus/journals/ijds/

Kannan, V. R., \& Tan, K. C. (2005). Just in time, total quality management, and supply chain management: understanding their linkages and impact on business performance. Omega, 33(2), 153-162. doi: 10.1016/j.omega.2004.03.012

Kaufman, J. C., Kaufman, S. B., Beghetto, R. A., Burgess, S. A., y Persson, R. S. (2009). Creative giftedness: beginnings, developments, and future promises. In International Handbook on Giftedness (pp. 585-598). Springer Netherlands.

Kaufman, J. C. (2010). Using creativity to reduce ethnic bias in college admissions. Review of General Psychology, 14(3), 189. doi: 10.1037/a0020133

Kaufman, J. C., Plucker, J. A., y Russell, C. M. (2012). Identifying and assessing creativity as a component of giftedness. Journal of Psychoeducational Assessment, 30(1), 60-73. doi: $10.1177 / 0734282911428196$

Kaufman, S. B., Reynolds, M. R., Liu, X., Kaufman, A. S., y McGrew, K. S. (2012). Are cognitive $g$ and academic achievement $g$ one and the same $g$ ? An exploration on the Woodcock-Johnson and Kaufman tests. Intelligence, 40(2), 123-138. doi: 10.1016/j.intell.2012.01.009

Kaufman, S. B. (2013). Ungifted: intelligence redefined. Perseus Books Group.

Kearns, H., Gardiner, M., \& Marshall, K. (2008). Innovation in PhD completion: The hardy shall succeed (and be happy!). Higher Education Research \& Development, 27(1), 7789. doi: 10.1080/07294360701658781 
Kim, K. H. (2005). Can only intelligent people be creative? A meta-analysis. Journal of Advanced Academics, 16(2-3), 57-66. doi: 10.4219/jsge-2005-473

Kim, K. H. (2006). Can we trust creativity tests? A review of the Torrance Tests of Creative Thinking (TTCT). Creativity research journal, 18(1), 3-14. doi: 10.1207/s15326934crj1801_2

Kim, K. H. (2008). Meta-Analyses of the Relationship of Creative Achievement to Both IQ and Divergent Thinking Test Scores. The Journal of Creative Behavior, 42(2), 106130. doi: 10.1002/j.2162-6057.2008.tb01290.x

Kuncel, N. Hezlett, S. y Ones, D. A. (2001). Comprehensive Meta-Analysis of the Predictive Validity of the Graduate Record Examinations: Implications for Graduate Student Selection and Performance. Psychological Bulletin 127(1), pp. 162-181. doi: 10.1037/0033-2909.127.1.162

Kuncel, N. R., Wee, S., Serafin, L., y Hezlett, S. A. (2010). The validity of the Graduate Record Examination for master's and doctoral programs: A meta-analytic investigation. Educational and Psychological Measurement, 70(2), 340-352. doi: $10.1177 / 0013164409344508$

Kuncel, N. \& Hezlett, S. (2007). Standardized tests predict graduate students' success. Science 315, 1080-1081. doi: 10.1126/science.1136618

Kuncel, N., Hezlett, S. y Ones, D. (2004). Academic Performance, Career Potential, Creativity, and Job Performance: Can One Construct Predict Them All? Journal of Personality and Social Psychology 86(1), 148-161. doi: 10.1037/0022-3514.86.1.148

Kyllonen, P., Walters, A. M., \& Kaufman, J. C. (2005). Noncognitive constructs and their assessment in graduate education: A review. Educational Assessment, 10(3), 153-184. doi: 10.1207/s15326977ea1003_2

Lohman, D. F. (2005). The role of nonverbal ability tests in identifying academically gifted students: An aptitude perspective. Gifted Child Quarterly, 49(2), 111-138. doi: $10.1177 / 001698620504900203$

López, M. y Flores, K. (2006). Análisis de competencias laborales a partir del Examen General para el Egreso de la Licenciatura (EGEL) y su relación con los cursos en línea. En actas del encuentro Virtual Educa. 
López, S. (2011). Visibilidad del conocimiento mexicano. La participación de las publicaciones científicas mexicanas en el ámbito internacional. Revista de la Educación Superior, 40(158), 151-165. Recuperado de http://publicaciones.anuies.mx/revista

Lovitts, B. E. (2005). Being a good course-taker is not enough: a theoretical perspective on the transition to independent research. Studies in Higher Education, 30(2), 137-154. doi: 10.1080/03075070500043093

Lovitts, B. E. (2008). The transition to independent research: who makes it, who doesn't, and why. The Journal of Higher Education, 79(3), 296-325. doi: 10.1353/jhe.0.0006

Márquez, A. M. (2012). El financiamiento de la educación en México Problemas y alternativas. Perfiles Educativos, 34, 107-117. Recuperado de http://www.iisue.unam.mx/perfiles/

Martínez, A., Urritia, M., Martínez, A. I., Ponce, A. y Gil, R. (2003). Perfil del estudiante de posgrado con éxito académico en la UNAM. Revista de investigación e innovación educativa, 32, 133-145. Recuperado de http://www.uam.es/servicios/apoyodocencia/ice/tarbiya/anteriores.html

Martínez, V.; Solís, L.; Osorio, E. (2000). Estudio sobre el procedimiento de selección de alumnos de nuevo ingreso, mediante el examen nacional EXANI-II y el aprovechamiento del nivel medio y superior, en la Facultad de Química de la UAEM. Ingreso de estudiantes de 1996, 1997, 1998 y 1999. En actas del tercer Congreso Internacional Retos y Expectativas de la Universidad. Coahuila, México.

Maya, R., Chávez, M., \& Apolinar, J. (2012). Posgrado en Ingeniería Química de la UMSNH. En Serna, R. y Pérez, R. (Coordinadores): Logros e innovación en el Posgrado, 170176. Consejo Mexicano de Estudios de Posgrado A.C.

Mazcorro, G., Aday, D., y Hernández, R. (2007). Una Evaluación estadística del examen de ingreso al posgrado EXANI-III en el proceso de admisión de la UPIICSA, IPN. Cuarto Seminario Internacional de Docencia Universitaria, CEDDES, Cienfuegos, Cuba.

Megginson, L. (2009). Noncognitive constructs in graduate admissions: An integrative review of available instruments. Nurse Educator, 34(6), 254-261. doi: 10.1097/NNE.0b013e3181bc7465

Montgomery, D. (2013). Gifted and talented children with special educational needs: Double exceptionality. Routledge.

Moon, B., y Leach, J. (2008). The power of pedagogy. Sage publications. 
Morales, R., Barrera, A. y Garnett, E. (2009). Validez predictiva y concurrente del EXANI-II, en la Universidad Autónoma del Estado de México. En memorias del X Congreso Nacional de Investigación Educativa (COMIE). Veracruz, México.

Muammar, O.M. (2011). Intelligence and self-control predict academic performance of Gifted on Non-gifted students. Asia-Pacific Journal of Gifted and talented Education, 3(1), 18-32. Recuperado de http://www.apfgifted.org/index.php/Index/publications

Oaten, M., \& Cheng, K. (2006). Longitudinal gains in self-regulation from regular physical exercise. British Journal of Health Psychology, 11, 717-733. doi: $10.1348 / 135910706 \times 96481$

Pasternak, R. (2013). Discipline, learning skills and academic achievement. Journal of Arts and Education, 1(1), pp. 1-11. Recuperado de http://www.accessinterjournals.org/jae/

Pekrun, R., Elliot, A. J., y Maier, M. A. (2009). Achievement goals and achievement emotions: Testing a model of their joint relations with academic performance. Journal of Educational Psychology, 101(1), 115. doi: 10.1037/a0013383

Pérez, R., Serna, M y. Barriga, C. (2012). Posgrados institucionales: Colaboraciones exitosas. En Serna, R. y Pérez, R. (Coordinadores): Logros e innovación en el Posgrado, 177 183. Consejo Mexicano de Estudios de Posgrado A.C.

Phillips, D. C., Barrow, R., Carr, D., Pring, R., Williams, I., Martin, J. y Martin, N., Bonnett, M. (Eds.). (2010). The SAGE Handbook of Philosophy of Education. SAGE publications.

Plucker, J. A., Beghetto, R. A., y Dow, G. T. (2004). Why isn't creativity more important to educational psychologists? Potentials, pitfalls, and future directions in creativity research. Educational Psychologist, 39(2), 83-96. doi: 10.1207/s15326985ep3902_1

Ponce, T. y García, S. (2003). Proceso de selección de alumnos en la UAEMEX, validez predictiva. En actas del tercer Congreso Internacional Retos y Expectativas de la Universidad. Coahuila, México.

Poropat, A. E. (2009). A meta-analysis of the five-factor model of personality and academic performance. Psychological bulletin, 135(2), 322. doi: 10.1037/a0014996

Senko, C., Hulleman, C. S., y Harackiewicz, J. M. (2011). Achievement goal theory at the crossroads: Old controversies, current challenges, and new directions. Educational Psychologist, 46(1), 26-47. doi: 10.1080/00461520.2011.538646 
Sevilla, D., Martín, M. y Guillermo, M. (2009). Optimización del proceso de selección para incrementar la eficiencia terminal de los programas de posgrado de la facultad de educación de la Universidad Autónoma de Yucatán. En actas del XXIII Congreso Nacional de Posgrado y Expo Posgrado 2009 (pp. 169-178.). San Luis Potosí, México.

Smith, R. L., Maroney, K., Nelson, K. W., Abel, A. L., \& Abel, H. S. (2006). Doctoral programs: Changing high rates of attrition. The Journal of Humanistic Counseling, Education and Development, 45(1), 17-31. doi: 10.1002/j.2161-1939.2006.tb00002.x

Solís, R. (2009). Análisis de las diferencias entre los estudiantes que se gradúan y los no obtienen el grado en el posgrado en construcción de la Facultad de Ingeniería de la UADY. En Actas del Seminario sobre docencia en diseño construcción de la UADY. Mérida, Yucatán, México.

Spaulding, L. S., y Rockinson-Szapkiw, A. J. (2012). Hearing their voices: Factors doctoral candidates attribute to their persistence. International Journal of Doctoral Studies, 7 , 199-219. Recuperado de http://www.informingscience.us/icarus/journals/ijds/

Sternberg, R. J., Bonney, C. R., Gabora, L., Y Merrifield, M. (2012). WICS: A model for college and university admissions. Educational Psychologist, 47(1), 30-41. doi: $10.1080 / 00461520.2011 .638882$

Strayhorn, T. L. (2014). What Role Does Grit Play in the Academic Success of Black Male Collegians at Predominantly White Institutions? Journal of African American Studies, 18(1), 1-10. doi: 10.1007/s12111-012-9243-0

Terman, L. M. (1917). The Stanford revision and extension of the Binet-Simon scale for measuring intelligence (Vol. 18). Warwick \& York, inc.

Terman, L. M., y Merrill, M. A. (1937). Measuring intelligence: A guide to the administration of the new revised Stanford-Binet tests of intelligence. Boston: Houghton Mifflin.

Tomsho, R. (2009, August 19). Adding personality to the college admissions mix. Wall Street Journal. Recuperado de http:// http://online.wsj.com/news/articles/SB10001424052970203612504574342732853413 584

Wao, H. O. y Onwuegbuzie, A. J. (2011). A mixed research investigation of factors related to time to the doctorate in education. International Journal of Doctoral Studies, 6, 115 134. Recuperado de http://www.informingscience.us/icarus/journals/ijds/ 
Was, C. (2006). Orientación de meta de logro academico: un nuevo planteamiento. Electronic Journal of Research in Educational Psychology, 4(3), 529-550. Recuperado de http://www.investigacion-psicopedagogica.org/revista/new/index.php

Watson, K. J., Blackstone, J. H., y Gardiner, S. C. (2007). The evolution of a management philosophy: the theory of constraints. Journal of Operations Management, 25(2), 387402. doi: 10.1016/j.jom.2006.04.004 